\title{
Pro-apoptotic Sorafenib signaling in murine hepatocytes depends on malignancy and is associated with PUMA expression in vitro and in vivo
}

\author{
R Sonntag ${ }^{1}$, N Gassler ${ }^{2}$, J-M Bangen ${ }^{1}$, C Trautwein ${ }^{1}$ and C Liedtke ${ }^{*, 1}$
}

The multi-kinase inhibitor Sorafenib increases the survival of patients with advanced hepatocellular carcinoma (HCC). Current data suggest that Sorafenib inhibits cellular proliferation and angiogenesis and promotes apoptosis. However, the underlying pro-apoptotic molecular mechanisms are incompletely understood. Here we compared the pro-apoptotic and anti-proliferative properties of Sorafenib in murine hepatoma cells and syngeneic healthy hepatocytes in vitro and in animal models of HCC and liver regeneration in vivo. In vitro, we demonstrate that cell cycle activity and expression of anti-apoptotic Bcl-2 like proteins are similarly downregulated by Sorafenib in Hepa1-6 hepatoma cells and in syngeneic primary hepatocytes. However, Sorafenibmediated activation of caspase-3 and induction of apoptosis were exclusively found in hepatoma cells, but not in matching primary hepatocytes. We validated these findings in vivo by applying an isograft HCC transplantation model and partial hepatectomy (PH) in C57BL/6 mice. Sorafenib treatment activated caspase-3 and thus apoptosis selectively in small tumor foci that originated from implanted Hepa1-6 cells but not in surrounding healthy hepatocytes. Similarly, Sorafenib did not induce apoptosis after PH. However, Sorafenib treatment transiently inhibited cell cycle progression and resulted in mitotic catastrophe and enhanced non-apoptotic liver injury during regeneration. Importantly, Sorafenib-mediated apoptosis in hepatoma cells was associated with the expression of p53-upregulated-modulator-of-apoptosis (PUMA). In contrast, regenerating livers after PH revealed downregulation of PUMA and were completely protected from Sorafenib-mediated apoptosis. We conclude that Sorafenib induces apoptosis selectively in hepatoma cells but not in healthy hepatocytes and can additionally increase non-apoptotic hepatocyte injury in the regenerating liver.

Cell Death and Disease (2014) 5, e1030; doi:10.1038/cddis.2013.557; published online 30 January 2014

Subject Category: Cancer

Sorafenib is the only approved drug for the treatment of patients with unresectable hepatocellular carcinoma (HCC) at present. ${ }^{1,2}$ In a phase III double-blind randomized control trial Sorafenib treatment improved overall survival of patients with advanced HCC from 7.9 months in the placebo group to 10.7 months. ${ }^{3}$ Deregulated signaling pathways in HCC have been extensively reviewed ${ }^{4}$ and include activation of the Raf/ Ras/mitogen-activated protein kinase (MAPK) pathways and over-expression of pro-angiogenic factors such as VEGF and PDGF. Sorafenib is a multi-kinase inhibitor with reported activity against Raf-1, B-Raf, VEGFR2, PDGFR and c-Kit receptors, among other receptor tyrosine kinases and serine threonine kinase ${ }^{5}$ and thus acts as an anti-proliferative and an anti-angiogenic agent. The anti-tumor efficacy of Sorafenib in HCC was intensively studied in the two hepatoma cell lines PLC/PRF/5 and HepG2 in vitro and also in vivo using heterotopic xenotransplantation models. ${ }^{6}$ These studies revealed that Sorafenib prevents cell cycle progression by inhibiting MAPK and through downregulation of Cyclin D1.
The mitochondrial membrane permeability is predominantly controlled by a balanced expression of pro-apoptotic and anti-apoptotic proteins of the Bcl-2 (B-cell lymphoma) family. Anti-apoptotic Bcl-2 proteins such as Bcl-2, Bcl-XI and $\mathrm{Mcl}-1$ are responsible for the maintenance of mitochondrial membrane permeability by inhibiting pro-apoptotic proteins and Apaf-1. ${ }^{7}$ Sorafenib reduces $\mathrm{Mcl}-1$ expression, which is associated with the induction of apoptosis in some tumor cell lines. ${ }^{6,8}$ However, the pro-apoptotic properties of Sorafenib are incompletely understood.

Here, we aimed to evaluate the potential pro-apoptotic and anti-proliferative effects of Sorafenib on healthy hepatocytes. To this end we compared Sorafenib-dependent signaling pathways in malignant hepatoma cells versus healthy hepatocytes in a syngeneic background in vitro and in vivo. We demonstrate that Sorafenib similarly induces cell cycle arrest in hepatoma cells and in liver after partial hepatectomy $(\mathrm{PH})$ while it specifically induces apoptosis only in malignant hepatoma cells in vitro and in vivo. Interestingly, apoptosis

\footnotetext{
${ }^{1}$ Department of Medicine III, University Hospital Aachen, RWTH Aachen University, Aachen, Germany and ${ }^{2}$ Institute of Pathology, University Hospital Aachen, RWTH Aachen University, Aachen, Germany

*Corresponding author: C Liedtke, Department of Medicine III, University Hospital Aachen, RWTH Aachen, Pauwelsstrasse 30, D-52074 Aachen, Germany. Tel: +49 024180 89249; Fax: +49 024180 82455; E-mail: cliedtke@ukaachen.de

Keywords: hepatocellular carcinoma; syngeneic transplantation model; mitotic catastrophe; liver regeneration; partial hepatectomy

Abbreviations: HCC, hepatocellular carcinoma; h, hour(s); MAPK, mitogen-activated protein kinase; Bcl-2, B-cell lymphoma-2; PH, partial hepatectomy; PUMA, p53-upregulated-modulator-of-apoptosis; WT, wild type; EGF, epidermal growth factor; PCNA, proliferating cell nuclear antigen; ERK, extracellular-signalregulated kinase(s); TUNEL, terminal deoxynucleotidyl transferase dUTP nick-end labeling; Rb, retinoblastoma protein; BrdU, 5-bromo-2'-deoxyuridine

Received 14.8.13; revised 05.12.13; accepted 13.12.13; Edited by G Melino
} 
induction was associated with the robust expression of $p 53-$ upregulated-modulator-of-apoptosis (PUMA). We also show that Sorafenib may trigger non-apoptotic cell death in regenerating livers by features resembling mitotic catastrophe. Thus, our study provides novel mechanistic data highlighting the benefits and risks of Sorafenib treatment in patients with HCC.

\section{Results}

Sorafenib induces cell cycle arrest via suppression of MAPK pathway and G1/S phase cyclins and promotes apoptosis in Hepa1-6 cells. The present study aimed to examine differential effects of Sorafenib on healthy hepatocytes versus malignant transformed hepatoma cells. All experiments were performed in a syngeneic murine system using either the mouse hepatoma cell line Hepa1-6 (derived from C57/LJ mice ${ }^{9}$ ) or C56BL/6 mice.

First, we determined the dose-dependent effects of Sorafenib on cell cycle progression in Hepa1-6 cells in vitro. Gene and protein expression analysis revealed that Sorafenib strongly inhibited cell cycle signaling in Hepa1-6 cells. Low Sorafenib concentrations $(\geq 5 \mu \mathrm{M})$ resulted in a $50 \%$ downregulation of Cyclin E1 gene expression and moderate inhibition of Cyclin D1 and Cyclin A2 transcription (Figure 1a). On the protein level, Sorafenib concentrations $\geq 10 \mu \mathrm{M}$ completely abrogated cyclin D1 and substantially reduced the expression of cyclins $E 1$ and $A 2$, respectively (Figure 1b). This was associated with the lack of Retinoblastoma protein $(\mathrm{Rb})$ phosphorylation and the downregulation of proliferating cell nuclear antigen (PCNA, Figure 1b). The onset of cell cycle is usually associated with the activation of Ras/Raf signaling involving phosphorylation of MAPK kinases (MEK1/2) and Extracellular-signal-regulated kinases (Erk1/2). Sorafenib dose-dependently inhibited phosphorylation of MEK1/2 and Erk1/2, respectively (Figure 1b), suggesting that Sorafenib blocks several steps of the Ras/Raf/MAPK pathway in Hepa1-6 cells.

We next studied time-dependent effects of Sorafenib on Hepa1-6 cells using an effective dose of $10 \mu \mathrm{M}$. Interestingly, Cyclin E1 gene expression was markedly downregulated already $4 \mathrm{~h}$ after Sorafenib treatment, which was not the case for Cyclin A2 and Cyclin D1 (Figure 1c). Altogether, these data suggest that Sorafenib-dependent inhibition of cell cycle signaling is mediated through several independent transcriptional and post-translational mechanisms in Hepa1-6 cells.

In order to verify that Sorafenib-mediated downregulation of cell cycle signaling results in true cell cycle arrest of Hepa1-6 cells, we measured the incorporation of the nucleoside analog
BrdU as a real-time indicator of DNA synthesis (S-phase, Figures $1 d$ and e). In good agreement with our protein studies, S-phase was slightly reduced by moderate Sorafenib doses $(\leq 10 \mu \mathrm{M})$, while stronger concentrations resulted in marked inhibition of DNA synthesis. Flow cytometry of propidium iodide (PI)-stained Hepa1-6 cells confirmed these findings and further revealed that increasing amounts of Sorafenib lead to the accumulation of Hepa1-6 cells in a stage with $4 n$ DNA content, indicative of G2/M-phase arrest (Figure 1f).

In addition, these FACS experiments demonstrated the formation of apoptotic cells with Sorafenib concentrations $\geq 10 \mu \mathrm{M}$ due to the appearance of a sub-G1 cell population. Of note, the apoptotic cell fraction was $\sim 20 \%$ of total cells after $50 \mu \mathrm{M}$ Sorafenib treatment (Figure 1f). This prompted us to investigate the pro-apoptotic signaling of Sorafenib in more detail. Within $24 \mathrm{~h}$, Sorafenib concentrations $\geq 10 \mu \mathrm{M}$ resulted in a dose-dependent reduction of cell density, change of morphology and obvious cell death (Figure 2a). Akt-Kinase activity promotes pro-survival/proliferative and anti-apoptotic pathways on transcriptional and post-translational levels, ${ }^{10}$ and was dose-dependently inhibited by Sorafenib (Figure 2b). In addition, Sorafenib inhibited phosphorylation of the Akt target Mdm2, reduced protein levels of the anti-apoptotic mediators such as $\mathrm{Mcl}-1, \mathrm{Bcl}-2$ and $\mathrm{Bcl}-\mathrm{XI}$ and promoted strong activation of the pro-apoptotic caspase-3 (Figure 2b), which resulted in the induction of apoptosis as evidenced by TUNEL-staining (Figure 2c). Thus, Sorafenib mediates pro-apoptotic and anti-proliferative effects in murine Hepa16 hepatoma cells.

\section{Sorafenib abrogates MAPK signaling and cell cycle} onset in resting and mitogen-activated primary hepatocytes. We aimed to investigate whether Sorafenib acts similarly on hepatoma cells and healthy hepatocytes of the same genetic origin and thus repeated our previous experiments in primary hepatocytes isolated from C57/BL6 wild-type (WT) mice with increasing concentrations of Sorafenib. Resting, non-stimulated hepatocytes initiate slight DNA replication within $24 \mathrm{~h}$ after ex vivo isolation, as indicated by phosphorylation of Rb, Erk1/2 and subsequent onset of PCNA expression (Figure 3a). Similar to our findings in Hepa1-6 cells, Sorafenib treatment abrogated cell cycle signaling at concentrations $\geq 20 \mu \mathrm{M}$ (Figure 3a), suggesting a similar anti-proliferative effect of Sorafenib in hepatocytes and hepatoma cells. Of note, high Sorafenib dosage also inhibited the expression of total Erk1/2 specifically in hepatocytes, but not in Hepa1-6 cells (compare Figure 1b).

To induce a strong proliferative response that better mimics the continuous proliferation of hepatoma cells, we

Figure 1 Sorafenib inhibits cell cycle progression in murine Hepa1-6 hepatoma cells. ( $(\mathbf{a}$ and $\mathbf{b}, \mathbf{d}-\mathbf{f}$ ) Hepa1-6 cells were treated with increasing concentrations of Sorafenib as indicated and analyzed $24 \mathrm{~h}$ after treatment. w/o, cells without treatment. (a) Gene expression of Cyclins A2, D1, E1 determined by qPCR. (b) Cell cycle signaling and MEK/Erk1/2 activation were analyzed by immunoblots. Expression of $\beta$-actin, total Erk1/2 and GAPDH were used as internal loading controls. (c) Hepa1-6 cells were treated with $10 \mu \mathrm{M}$ Sorafenib, DMSO alone or left untreated (w/o) and analyzed at indicated time points after treatment for gene expression of Cyclins A2, D1 and E1. (d and e) Hepa1-6 cells were analyzed for BrdU incorporation by fluorescence microscopy. (d) Representative images are shown. BrdU is stained in green (arrows) indicating DNA synthesis. Total nuclei are counter-stained with DAPI (blue). Control: cells treated with DMSO only. (e) Quantification of experiments shown in (d). A minimum of 10 high-magnification fields $(\times 200)$ per condition were quantified. ${ }^{* \star} P<0.01 ;{ }^{* * *} P<0.001$. (f) Cells were stained with propidium iodide and subjected to FACS analysis. For better comparison, representative plots with an overlay of control cells (DMSO control, black line) and respective treatment groups (red) are shown. Sub-G1 populations with DNA content $<2 n$ indicate apoptotic cells and were compared with controls 
co-stimulated primary hepatocytes with increasing concentrations of Sorafenib and the mitogens epidermal growth factor (EGF) and insulin. We recently demonstrated that under these conditions WT primary hepatocytes start to express Cyclin A2 - and thus DNA replication - $48 \mathrm{~h}$ after EGF/insulin treatment and show maximal expression after $\sim 3$ days. ${ }^{11}$ We thus focused on Sorafenib-mediated effects 48 and $72 \mathrm{~h}$ post EGF/insulin treatment as this is apparently a

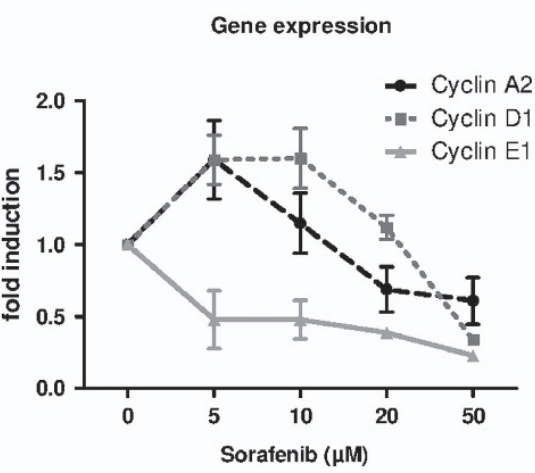

d

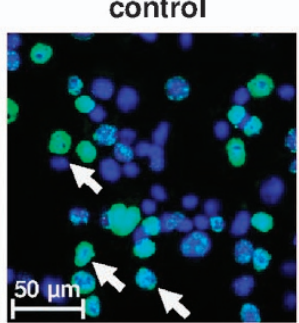

b
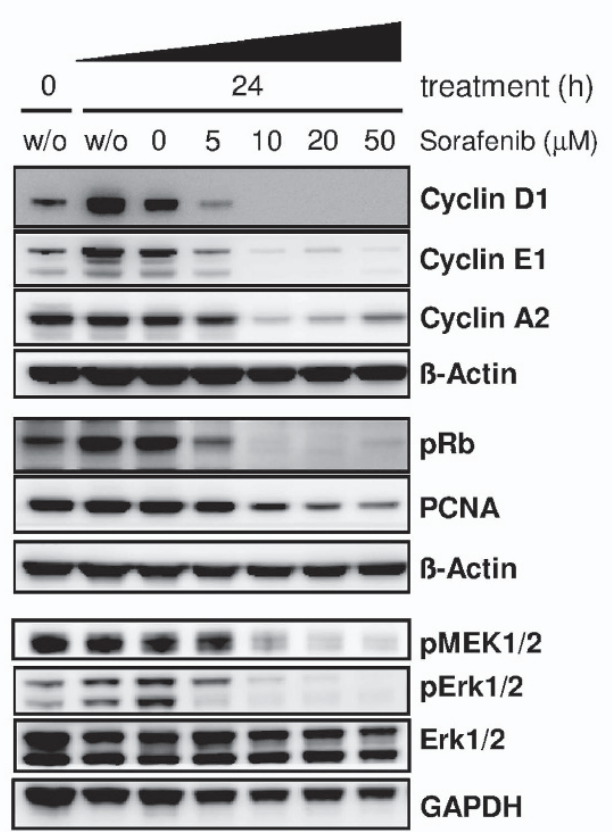

Cell cycle in hepatoma cells

GAPDH
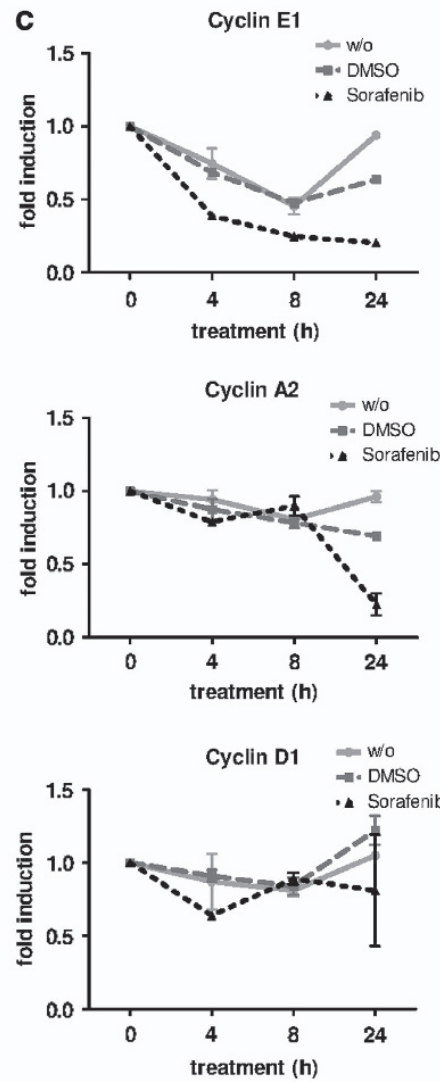
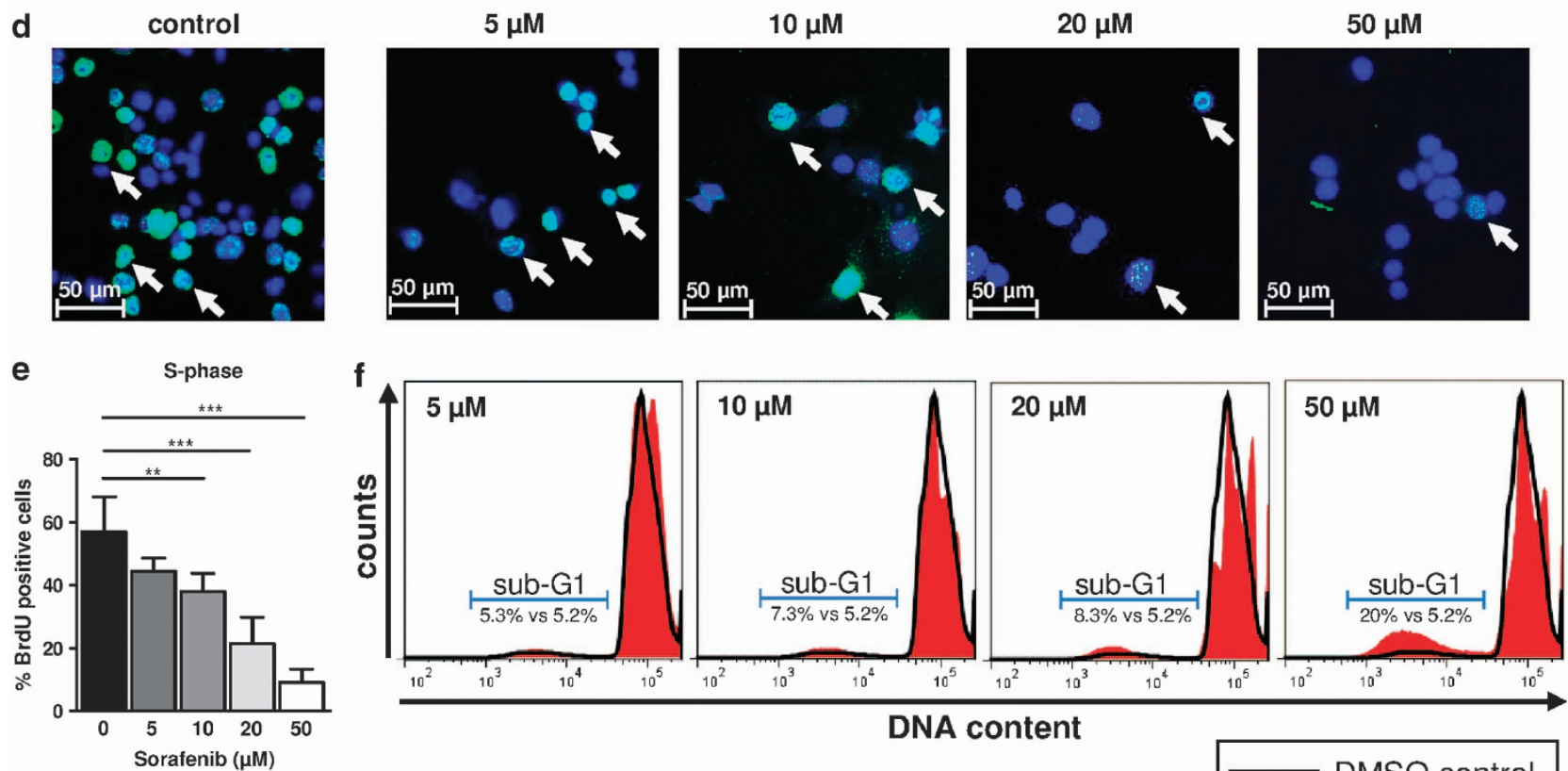

DNA content

DMSO control Sorafenib 
a
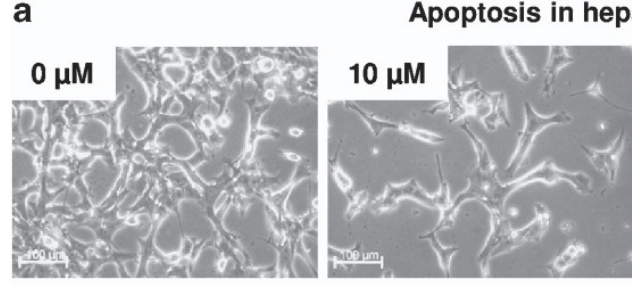

b

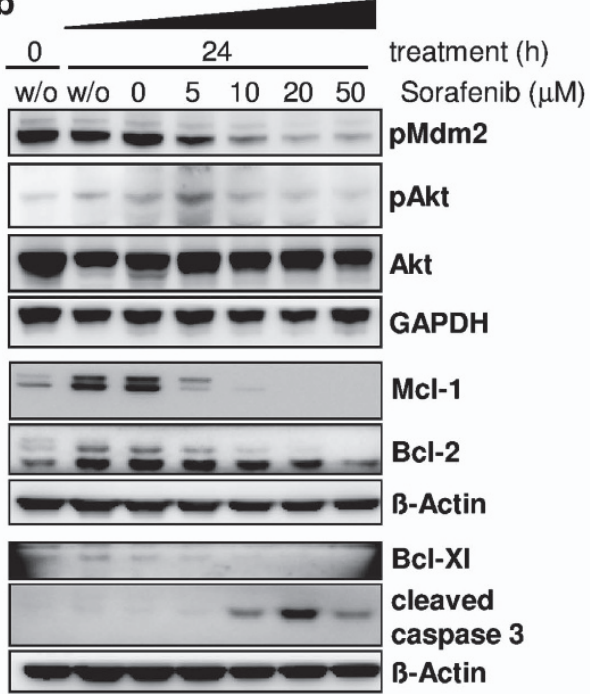

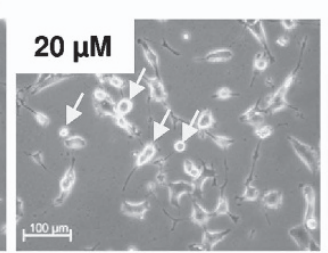

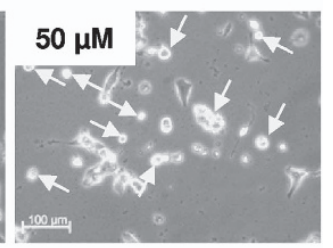

c
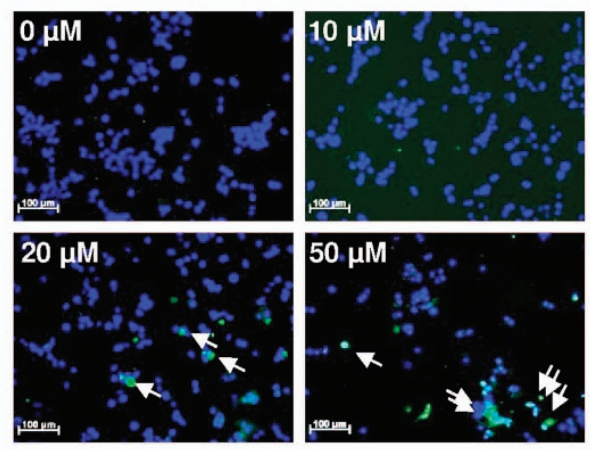

Figure 2 Sorafenib triggers apoptosis in murine Hepa1-6 hepatoma cells. Hepa1-6 cells were treated with increasing concentrations of Sorafenib as indicated and analyzed $24 \mathrm{~h}$ after treatment. w/o, cells without treatment. (a) Cell morphology of Hepa1-6 cells after treatment with increasing concentrations of Sorafenib as indicated. Cells were analyzed $24 \mathrm{~h}$ after treatment by phase contrast microscopy. Arrows highlight dying cells. (b) Immunoblots for Mcl-1, Bcl-2, Bcl-XI, pMdm2 (anti-apoptotic), cleaved caspase-3 (pro-apoptotic) and phosphorylated Akt (pro-survival). Total Akt, $\beta$-actin and GAPDH were used as internal loading controls. (c) TUNEL-staining of apoptotic Hepa1-6 cells (green, arrows). Blue, counter-staining of total nuclei with DAPI

the time frame of maximal DNA replication in mitogenstimulated hepatocytes. On the transcriptional level, the kinetics of Cyclin A2 induction without Sorafenib treatment were in good agreement with our previous data. ${ }^{11}$ Low doses of Sorafenib $(5 \mu \mathrm{M})$ delayed Cyclin A2 induction but still allowed full induction $72 \mathrm{~h}$ post treatment (Figure $3 \mathrm{~b}$ ). In contrast, Cyclin A2 gene induction was completely blocked by Sorafenib at concentrations $\geq 20 \mu \mathrm{M}$ (Figure $3 \mathrm{~b}$ and Supplementary Figure 1A). Hence, Sorafenib repressed mitogen signaling ( $p$ Erk1/2) and mediators for S-phase (Cyclin A2, PCNA, pRb) and mitosis (Cyclin B1) posttranscriptionally (Figures $3 c$ and $d$ ). Inhibition of S-phase and cell cycle arrest was confirmed by BrdU incorporation analysis (Figures $3 e$ and $f$, Supplementary Figure 1B). EGF/ insulin stimulation alone resulted in sustained DNA synthesis in primary hepatocytes, which was substantially reduced by Sorafenib concentrations $\geq 20 \mu \mathrm{M}$. Altogether, Sorafenib similarly inhibits cell cycle initiation in hepatoma cells and non-transformed hepatocytes.

\section{Sorafenib specifically induces caspase-3-mediated apoptosis in malignant transformed hepatocytes in vitro and in vivo. We next tested the pro-apoptotic properties of Sorafenib on ex vivo isolated primary hepato- cytes. Following Sorafenib treatment, resting primary hepatocytes showed a dose-dependent reduction of the}

anti-apoptotic proteins phospho-Mdm2, Bcl-2 and Mcl-1 and inhibition of Akt-Kinase activity similar to hepatoma cells (Figure 4a, Supplementary Figure 1c). However, in contrast to Hepa1-6 cells, we did not detect any proteolytic activation of caspase-3 in primary hepatocytes (Figure 4a), suggesting that the reduction of anti-apoptotic signaling alone is not capable of inducing apoptosis in resting hepatocytes.

We thus tested whether Sorafenib might induce apoptosis in mitogen-activated cells. Therefore, we co-stimulated Sorafenib-treated primary hepatocytes with EGF/insulin and analyzed the apoptotic response 48-72 h after treatment. As observed in hepatoma cells and resting hepatocytes, the anti-apoptotic proteins $\mathrm{Bcl}-\mathrm{Xl}$ and $\mathrm{Bcl}-2$ were downregulated and phosphorylation of the pro-survival factors pMdm2 and pAkt were inhibited in a dose-dependent manner (Figures $4 b$ and c). However, mitogenic stimulation did not trigger Sorafenib-mediated caspase-3 activation as investigated by immunoblot and caspase-3 enzyme activity assays (Figures $4 \mathrm{c}$ and $\mathrm{d}$ ), suggesting that Sorafenib mediates apoptosis specifically in malignant transformed hepatocytes.

To test this hypothesis in vivo we took advantage of the immunocompatibility between C57/BL6 mice and Hepa1-6 cells. We injected $3 \times 10^{6}$ Hepa1-6 cells i.v. into 8-week-old C57/BL6 mice (Figure 5a). After 3 weeks small hepatic tumor foci of $\sim 50-100 \mu \mathrm{m}$ diameter were found, which were characterized by high cellular density and strong proliferation 


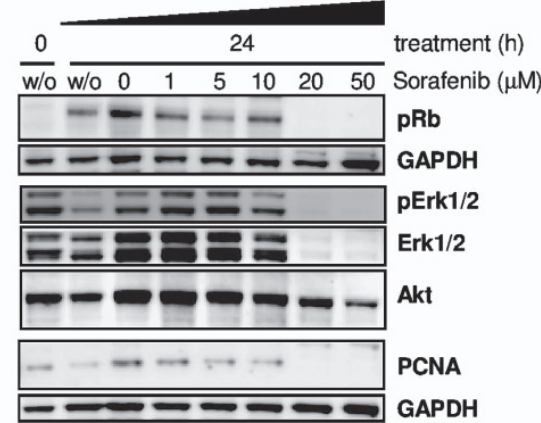

Cell cycle in hepatocytes

b

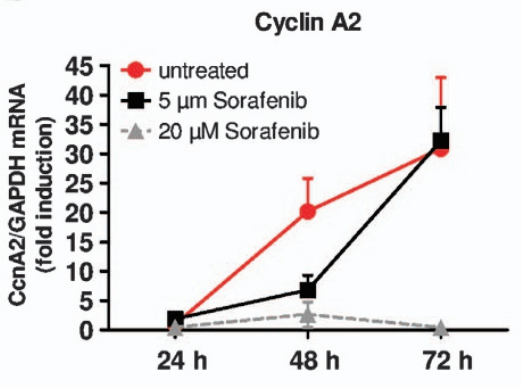

C

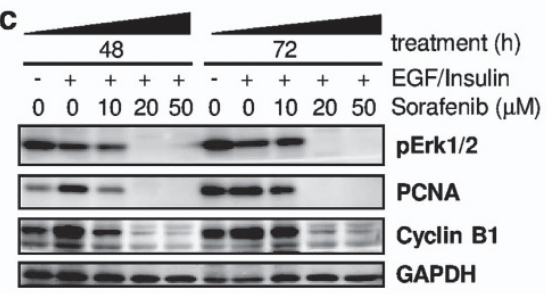

d

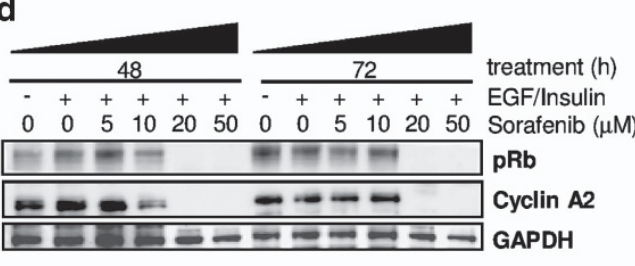

e

48 h EGF/insulin/Sorafenib
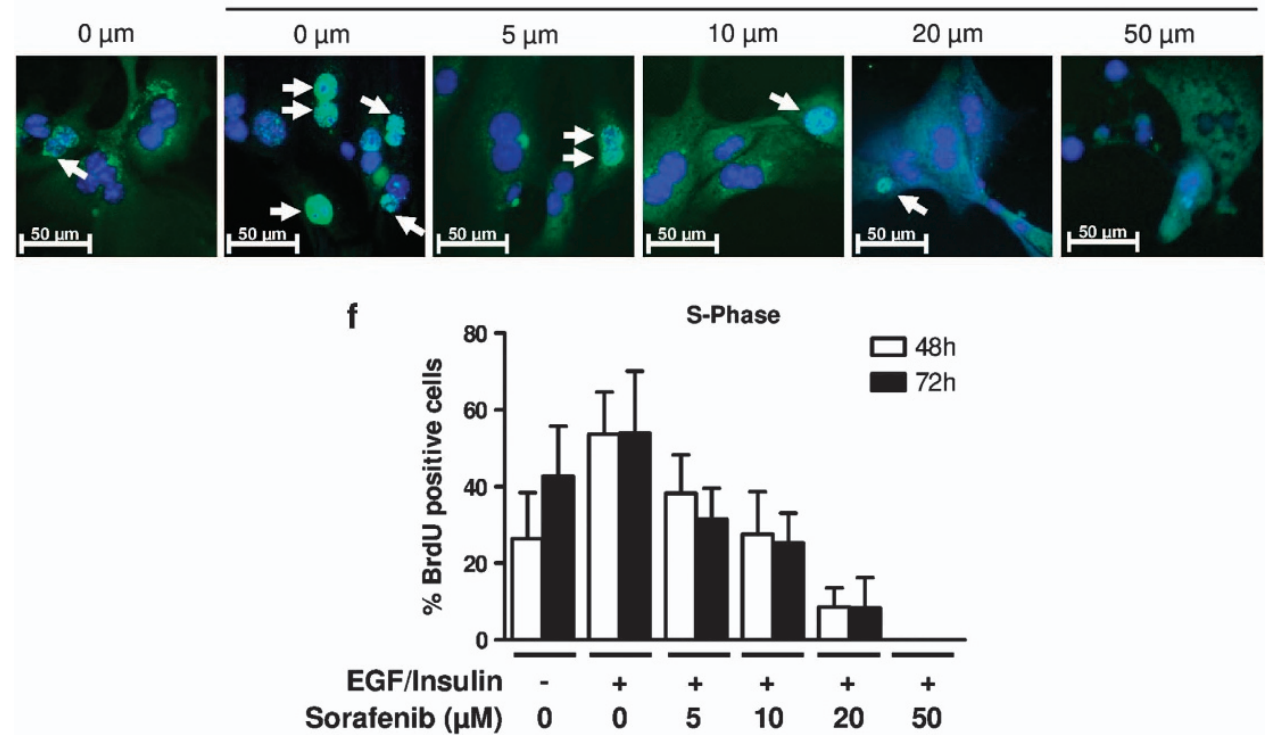

Figure 3 Sorafenib inhibits cell cycle progression in resting and proliferating primary hepatocytes. Primary hepatocytes were treated with increasing concentrations of Sorafenib, the solvent DMSO alone $(0 \mu \mathrm{m})$ or left untreated (w/o). (a) Protein expression of phosphorylated Rb (pRb), phosphorylated Erk1/2 (pErk1/2) and PCNA. The expression levels of GAPDH, total Erk1/2 and total Akt were used as internal controls. (b-f) Primary hepatocytes were mitogen-stimulated with EGF and insulin and analyzed after 48 and $72 \mathrm{~h}$. (b) Cyclin A2 mRNA expression in response to increasing concentrations of Sorafenib. (c) Protein expression of pErk1/2, Cyclin B1 and PCNA. (d) Immunoblot analysis of Rb phosphorylation and Cyclin A2 expression. (e-f) Primary hepatocytes were analyzed for BrdU incorporation by fluorescence microscopy after $48 \mathrm{~h}$ of mitogen stimulation. (e) Representative images are shown. BrdU-positive nuclei are stained in green (arrows); total nuclei are counter-stained with DAPI (blue). Owing to green autofluorescence, morphology of hepatocytes is visible. Controls $(0 \mu \mathrm{M}$, left) did not receive mitogens. (f) Quantification of experiments shown in (e). A minimum of 10 high magnification fields $(\times 200)$ per condition were quantified

as evidenced by nuclear Ki-67 expression (Figures $5 \mathrm{~b}$ and $\mathrm{c}$ ). A cohort of these mice was treated with Sorafenib or solvent for 4 consecutive days and analyzed for intrahepatic caspase3 activation. Only Sorafenib-treated livers revealed focal caspase-3 activation within engrafted tumor foci, while surrounding resident hepatocytes did not undergo any apoptotic cell death (Figures $5 \mathrm{~d}$ and e). These data demonstrated highly specific pro-apoptotic properties of Sorafenib in malignant transformed hepatocytes in vivo.

\section{Sorafenib inhibits hepatic cell cycle progression in vivo} after PH. Our previous experiments demonstrated that Sorafenib similarly inhibited cell cycle in healthy and transformed hepatocytes in vitro but did not induce apoptosis 

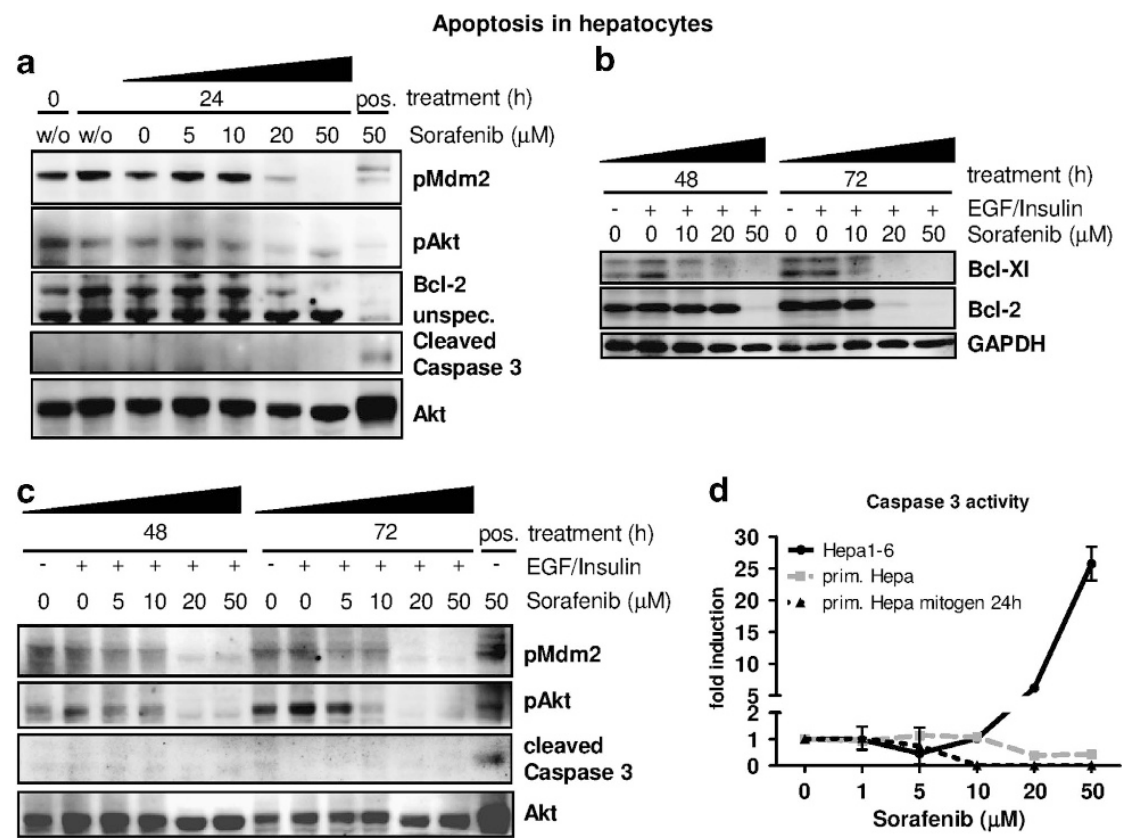

Figure 4 Resting and proliferating primary hepatocytes are resistant to Sorafenib-mediated apoptosis. Pro and anti-apoptotic signals were analyzed under resting and mitogen conditions. (a) Primary hepatocytes were treated with increasing concentrations of Sorafenib, the solvent DMSO alone $(0 \mu \mathrm{m})$ or left untreated (w/o). Immunoblot analysis of Mdm2 activation (pMdm2), Akt-phosphorylation (pAkt), Bcl-2 and cleaved (activated) caspase-3 is shown. Positive control (pos): Hepa1-6 cells stimulated with $50 \mu \mathrm{M}$ Sorafenib for $24 \mathrm{~h}$. Total Akt was determined as internal loading control. (b-d) Primary hepatocytes were co-stimulated with EGF and insulin and analyzed after 48 or $72 \mathrm{~h}$, respectively. (b) Immunoblot analysis of Bcl-2 and Bcl-XI. (c) Protein levels of pMdm2, pAkt and cleaved caspase-3. Positive control (pos): Hepa1-6 cells stimulated with $50 \mu \mathrm{M}$ Sorafenib for $24 \mathrm{~h}$. Total Akt is shown as internal control. (d) Determination of specific caspase-3 enzyme activity in primary hepatocytes (prim. Hepa mitogen: EGF/ insulin/Sorafenib treatment for $24 \mathrm{~h}$; prim. Hepa: Sorafenib treatment only). Hepa1-6 cells were equally treated with Sorafenib for $24 \mathrm{~h}$. Activity (fluorescence units/ $\mu \mathrm{g}$ protein) was calculated as fold induction in comparison to DMSO-treated controls

of non-malignant hepatocytes. To further evaluate the relevance of this observation in vivo, we performed $\mathrm{PH}$ in WT mice that were orally treated with Sorafenib $2 \mathrm{~h}$ before and once daily after surgery (Figure 6a). Animals were killed at the peak of S-phase (48 host $\mathrm{PH})$ and after terminated regeneration ( $96 \mathrm{~h}$ post $\mathrm{PH})$.

$\mathrm{Ki}-67$ - a general marker for cell cycle activity - is expressed in S, G2 and M-phase. ${ }^{12}$ In agreement with earlier studies, $\sim 40 \%$ of hepatocytes expressed Ki-67 48 h after $\mathrm{PH}$. Interestingly, treatment with ethanol-containing vehicle alone already resulted in a $50 \%$ reduction of overall cell cycle activity $48 \mathrm{~h}$ after $\mathrm{PH}$. However, Sorafenib treatment further reduced the number of proliferating cells by $\sim 60 \%$ in comparison to solvent-treated mice. The inhibitory effect of Sorafenib was transient and restricted to $48 \mathrm{~h}$, but not $96 \mathrm{~h}$ post $\mathrm{PH}$ (Figures $6 \mathrm{~b}$ and $\mathrm{C})$. We demonstrated that Sorafenib already inhibited hepatic S-phase at the peak of DNA replication ( $48 \mathrm{~h}$ post $\mathrm{PH}$ ) as evidenced by BrdU incorporation analysis (Figures $6 \mathrm{~d}$ and e). To further validate these findings, we analyzed expression of cell cycle-specific proteins induced at G1/ S-phase transition ( $p R b, P C N A)$, S-phase progression (Cyclin $\mathrm{A2}$ ) and mitosis (Cyclin B1) in liver samples $48 \mathrm{~h}$ after $\mathrm{PH}$. Of note, the solvent alone already had a moderated effect on protein expression, while Sorafenib substantially downregulated all investigated proteins (Figure 6f, Supplementary Figure 2A). Strikingly, expression of the cell cycle inhibitor p21 was similar in Sorafenib- and vehicle-treated mice, suggesting that the observed cell cycle arrest in the Sorafenib group was p21-independent (Figure 6f). Interestingly, vehicle treatment inhibited hepatocyte mitosis as evidenced by histological analysis, which was not further impaired by Sorafenib despite marked Cyclin B1 reduction in this group (Figures $6 \mathrm{f}$ and $\mathrm{g}$ ).

Continuous Sorafenib uptake induces non-apoptotic liver injury in the regenerating liver through disordered mitosis. We next analyzed the consequences of Sorafenibmediated cell cycle arrest for the regenerating liver after $\mathrm{PH}$. Unexpectedly, $96 \mathrm{~h}$ after $\mathrm{PH}$, Sorafenib-treated mice showed normal liver mass reconstitution in comparison to hepatectomized mice with or without vehicle treatment (Figure 7a), which implies that Sorafenib does not block but rather delays the hepatic cell cycle or alternatively triggers liver mass reconstitution through hypertrophic cell growth of hepatocytes as reported earlier. ${ }^{13,14}$

In agreement with our previous data, we did not detect elevated caspase-3 enzyme activity exceeding background levels in any treatment group after $\mathrm{PH}$ (Figure 7b), further confirming that Sorafenib does not induce apoptosis in nonmalignant hepatocytes independent of their proliferation status. However, Sorafenib-treated mice revealed significantly elevated serum transaminase levels (ALT, AST) 48 and $96 \mathrm{~h}$ after $\mathrm{PH}$ (Figure 7c), indicating that Sorafenib triggers enhanced non-apoptotic hepatocyte injury in the regenerating liver, which was not associated with enhanced infiltration of granulocytes (Supplementary Figure 2B-C).

A thorough histological examination of regenerating livers revealed that Sorafenib treatment triggered aberrant 
a
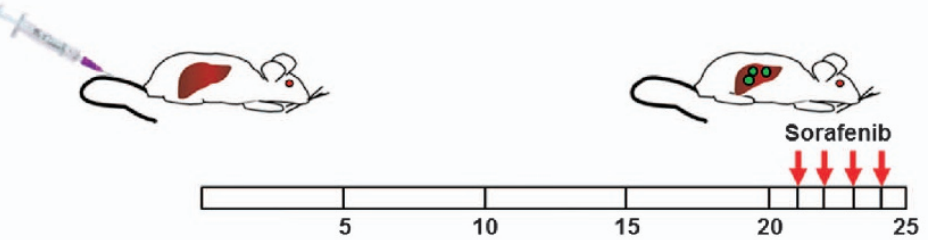

days after

implantation<smiles>[Te][Te]</smiles>

inject $3 \times 10^{6}$

Hepa1-6 cells i.v.

b
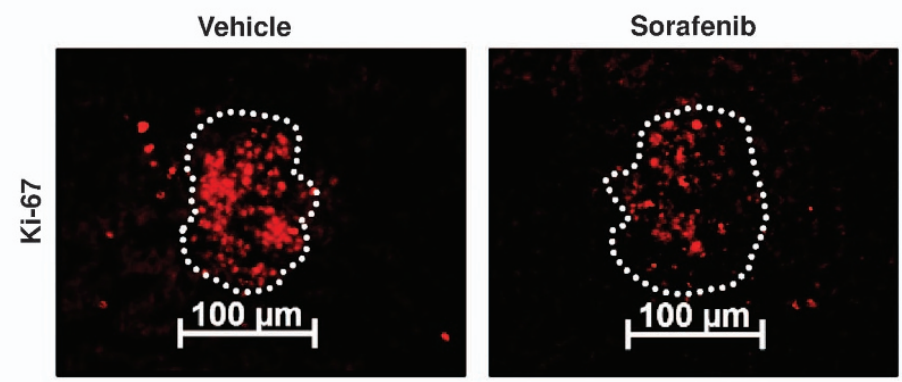

C
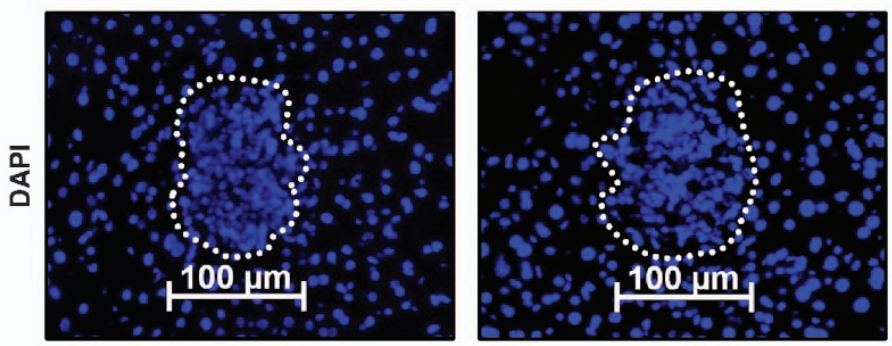

d
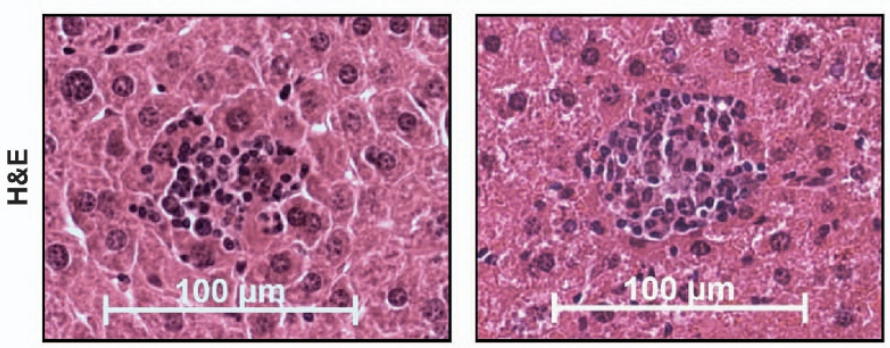

e
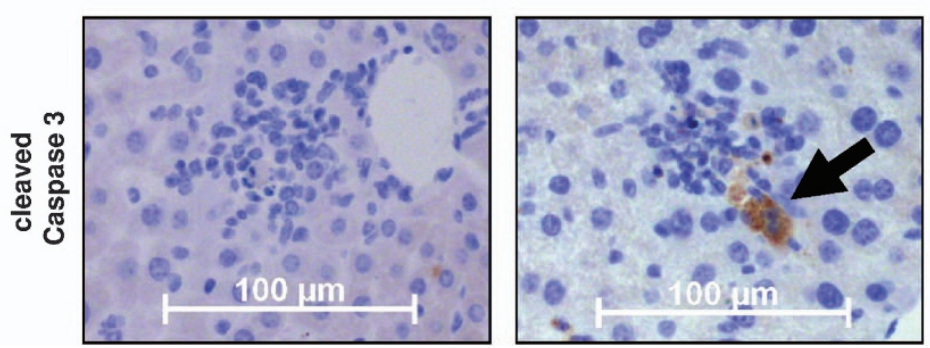

Figure 5 Sorafenib specifically induces apoptosis in hepatoma cells, but not in resting hepatocytes in vivo. (a) Experimental setup. WT mice were injected i.v. with $3 \times 10^{6}$ Hepa1-6 cells to induce hepatoma cell engraftment in liver. Three weeks after cell implantation, mice ( $n=4$ per group) were fed with Sorafenib or vehicle for 4 consecutive days and killed thereafter. Representative images are shown. (b) Ki-67 staining (red) of liver sections confirming strong proliferation of engrafted hepatoma cells. (c) Sections in (b) were counter-stained with DAPI (blue). Tumor areas appear as foci with high cellular density and are highlighted (white). (d) H\&E staining of liver sections showing engraftment of hepatoma cells. (e) Staining of activated caspase-3 (brown, arrow) indicating apoptosis

mitosis during liver regeneration. Regular hepatocyte mitosis is characterized by packed and condensed DNA along the equatorial plane, reflecting the transition from pro-/metaphase to anaphase (Figure 7d, left panel). We observed sixfold increased incidence of irregular mitosis, characterized by aberrant package of chromosomes, displaying bends, lobes and tripolarity. Additionally, we detected increased frequency of single hepatocytes with unpacked, free condensed DNA 
a Sorafenib Sorafenib Sorafenib Sorafenib Sorafenib
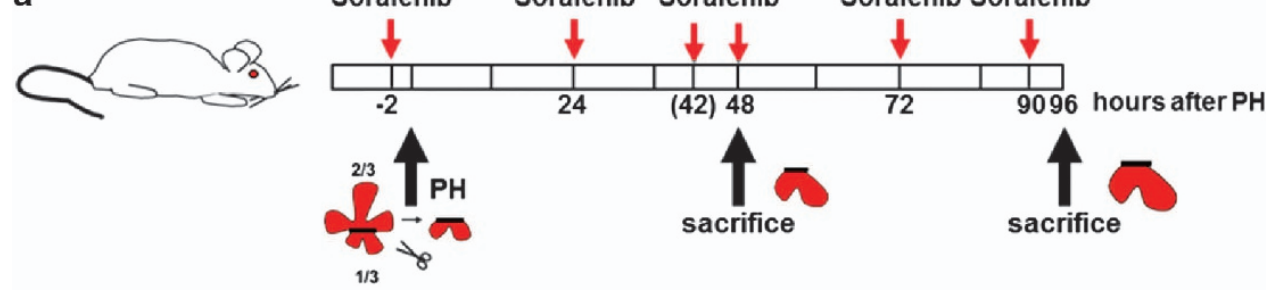

Cell cycle
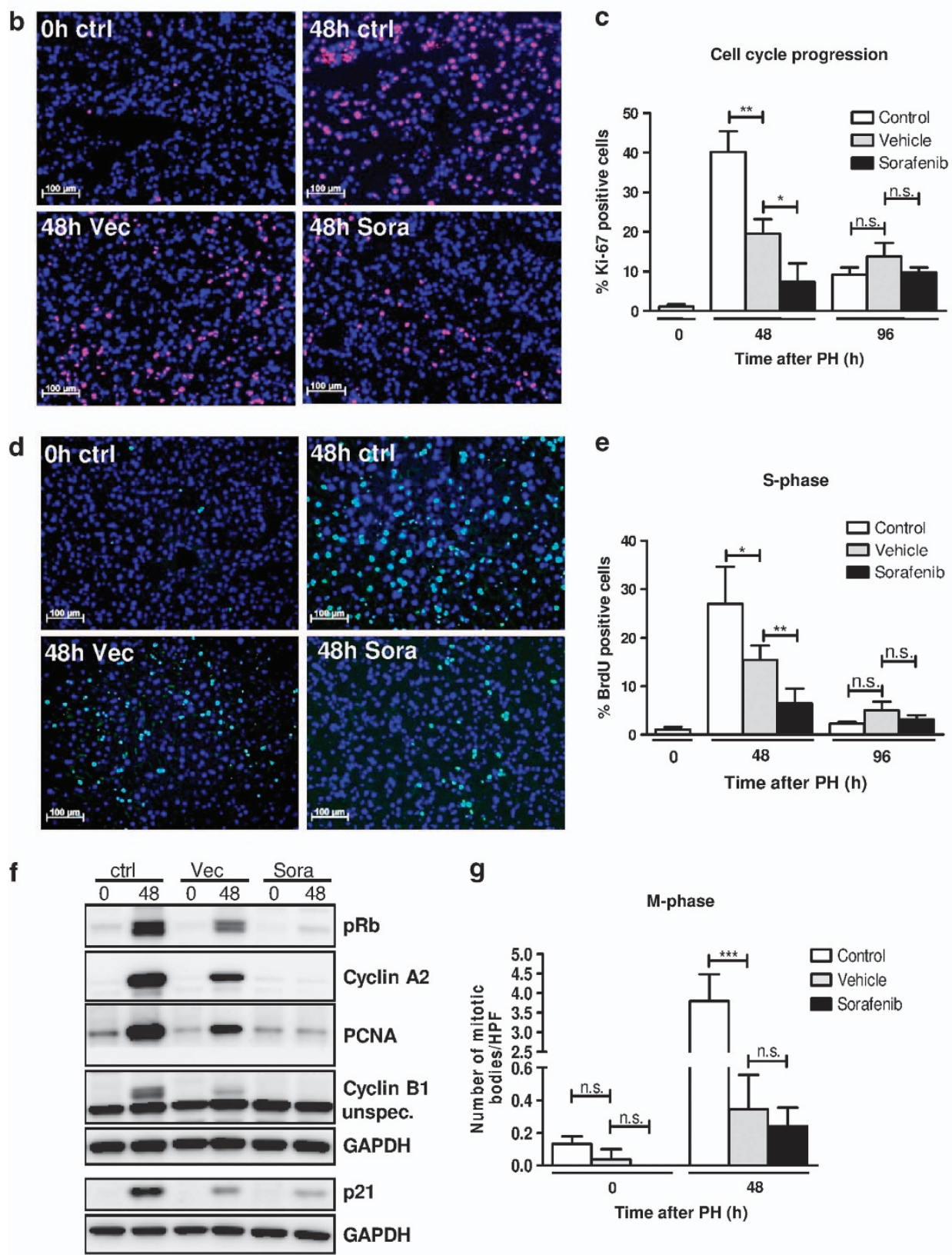

g

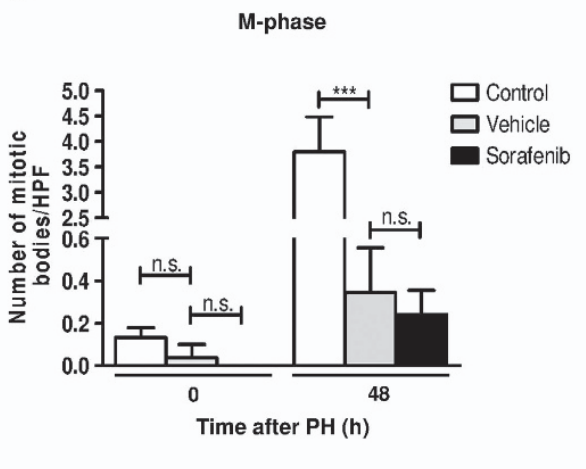

Figure 6 Sorafenib inhibits cell cycle progression in liver after PH and (a) Experimental setting. WT mice received Sorafenib or vehicle $2 \mathrm{~h}$ before PH and every 24 or $6 \mathrm{~h}$ before sacrificed (arrows). Controls were either left completely untreated ( $0 \mathrm{~h}$ ctrl) or subjected to PH only ( $48 \mathrm{~h}$ ctrl). (b) Ki-67 stainings (red) of liver sections $48 \mathrm{~h}$ after PH. Total nuclei are counter-stained with DAPI (blue). (c) Quantification of Ki-67 positive nuclei. (d) Determination of BrdU incorporation (green) $48 \mathrm{~h}$ after PH indicating DNA replication. Total nuclei are counter-stained with DAPI (blue). (e) S-phase progression was determined as the percentage of BrdU-positive nuclei. (f) Expression analysis of cell cycle-related proteins by immunoblot. (g) Quantification of mitotic bodies in H\&E-stained liver sections $48 \mathrm{~h}$ after $\mathrm{PH}$. ${ }^{*} P<0.05 ;{ }^{* *} P<0.01$; ${ }^{* \star \star} P<0.001$; n.s., not significant 
a

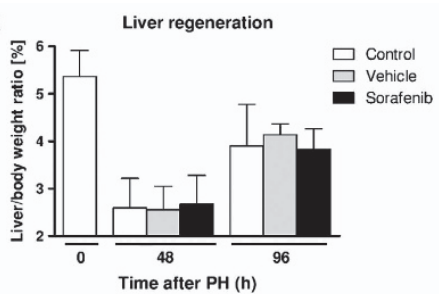

b

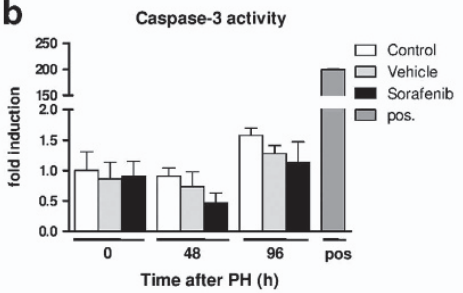

C

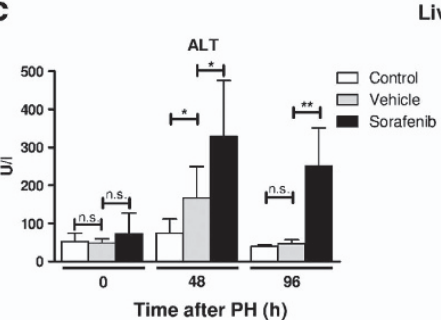

Liver injury
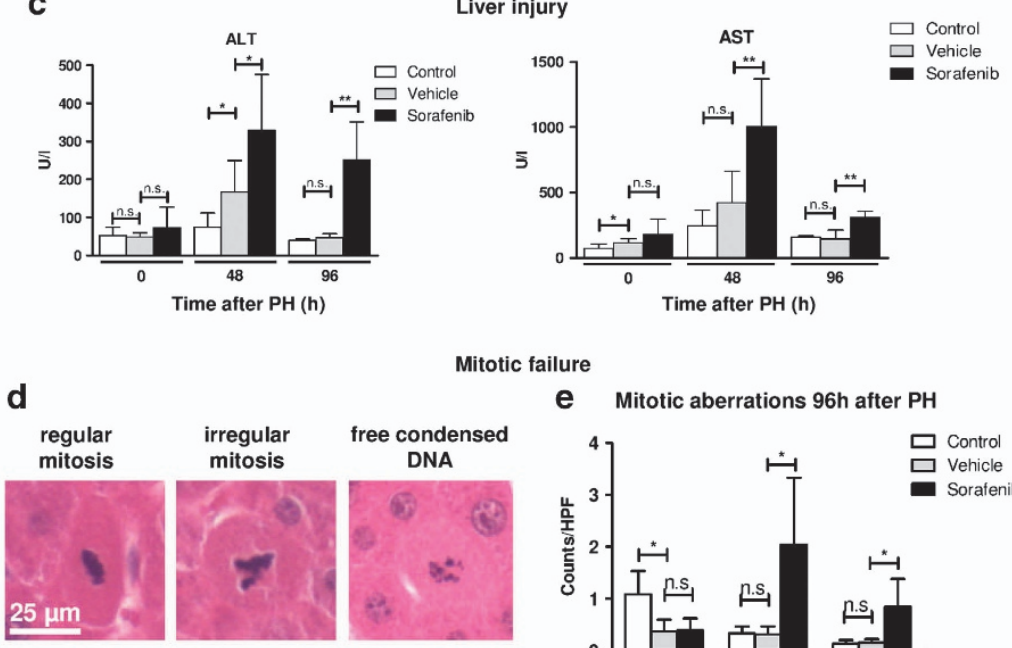

Mitotic failure

e Mitotic aberrations $96 \mathrm{~h}$ after $\mathrm{PH}$

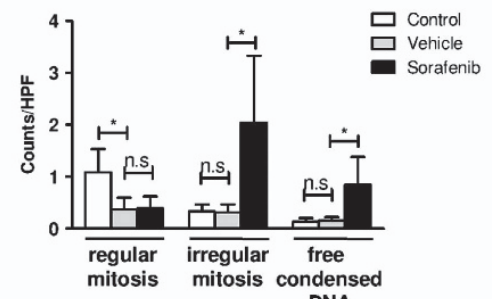

PUMA expression
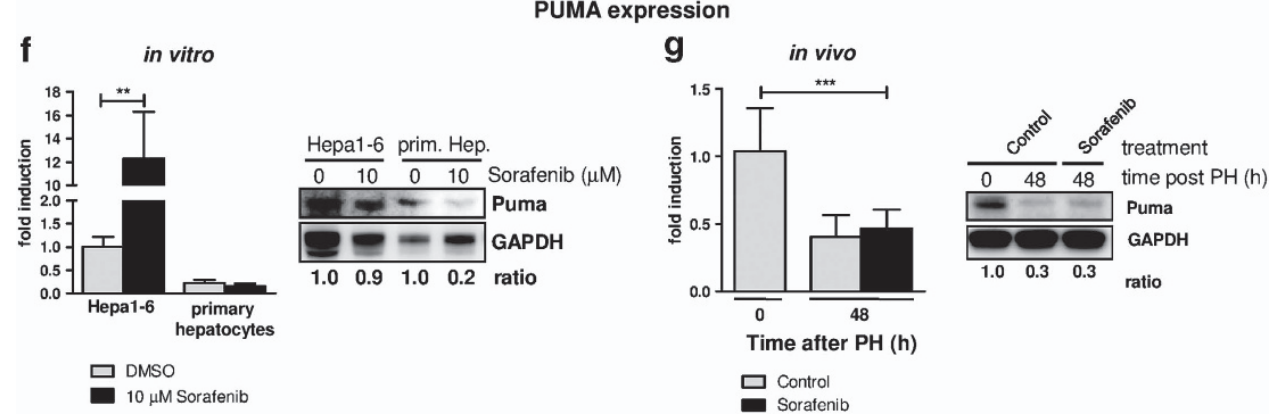

Figure 7 Sorafenib triggers increased liver injury and non-apoptotic hepatocyte death. WT mice were treated as shown in Figure 6a. At time points indicated, ex vivo isolated livers were analyzed for markers of liver injury and apoptosis. (a) Liver mass index at indicated time points after PH. (b) Caspase-3 enzyme activities of liver proteins after combined PH/Sorafenib or PH/vehicle treatments. Pos. control: Hepa1-6 cells treated with $50 \mu \mathrm{m}$ Sorafenib for $24 \mathrm{~h}$. (c) Serum levels of alanine aminotransferase (ALT) and aspartate aminotransferase (AST) indicative of overall liver injury. (d) Histology of mitotic hepatocytes after Sorafenib treatment $96 \mathrm{~h}$ post PH. Representative liver sections showing frequent irregular mitotic bodies and free condensed cytoplasmic DNA. (e) Quantitative analysis of aberrant mitosis. HPF: $\times 200$ magnification field. (f) Gene and protein expression of PUMA in vitro. Hepa1-6 cells and primary hepatocytes were stimulated with $10 \mu \mathrm{M}$ Sorafenib or DMSO for $24 \mathrm{~h}$. Left, gene expression determined by qPCR. Right, immunoblot analysis of PUMA protein. Signal intensities were quantified using digital densitometry and calculated as PUMA/GAPDH ratio. (g) Gene and protein expression of PUMA in vivo. PUMA expression was analyzed in Sorafenib- or vehicle (control)-treated livers $48 \mathrm{~h}$ after PH. Left, gene expression determined by qPCR. Right, immunoblot analysis and densitometric quantification. ${ }^{*} P<0.05 ;{ }^{*} P<0.01 ;{ }^{* \star *} P<0.001$; n.s., not significant

fragments throughout the cytoplasm in Sorafenib-treated livers (Figures $7 d$ and e). Thus, Sorafenib treatment does not induce apoptosis in the regenerating liver but leads to changes in mitosis progression and non-apoptotic liver injury.

We finally addressed the important question how Sorafenib induces apoptosis selectively in malignant hepatoma cells. Recent studies indicated that in colon carcinoma cells Sorafenib-mediated apoptosis was associated with the induction of PUMA. ${ }^{15}$ We thus measured PUMA gene and protein expression in stimulated hepatoma cells, primary hepatocytes and Sorafenib-treated mice after $\mathrm{PH}$, respectively. On the transcriptional level, PUMA gene expression was substantially higher in hepatoma cells and even further induced after Sorafenib treatment when compared with hepatocytes. Immunoblot analysis revealed robust PUMA protein expression in Hepa1-6 cells with or without Sorafenib treatment but clear decrease of PUMA levels in Sorafenibtreated hepatocytes (Figure 7f). Following PH, PUMA was 
significantly downregulated in Sorafenib-treated mice $48 \mathrm{~h}$ after $\mathrm{PH}$ in both control and Sorafenib-treated groups (Figure 7g). Altogether, protection from Sorafenibdriven apoptosis was found exclusively in non-malignant hepatocytes with low PUMA levels in vitro and in vivo.

\section{Discussion}

The multi-kinase inhibitor Sorafenib is the only approved systemic therapy for patients with advanced HCC so far and results in significant but modest overall survival. ${ }^{3,16,17}$ Although it is meanwhile well accepted that Sorafenib mediates pleiotropic effects by promoting apoptosis and inhibiting cellular proliferation and angiogenesis, the precise molecular mechanisms are still incompletely understood. For technical and ethical reasons it is obvious that the cellular and molecular effects of Sorafenib cannot be thoroughly analyzed in patients. The majority of previous mechanistic data on the anti-tumor activity of Sorafenib therefore comes from studies in human hepatoma cell lines or from xenograft models in mice and rats. ${ }^{6,17,18}$ However, these approaches have limitations as they only incompletely reflect the situation in a human liver with advanced HCC, cirrhosis and liver regeneration.

Here, we performed for the first time a comprehensive study of Sorafenib-mediated effects in a homogenous system using mice, primary (healthy) hepatocytes and malignant hepatoma cells with a syngeneic genetic background allowing comparative analyses in vivo and in vitro. We demonstrate that Erksignaling, cell cycle activity and expression of anti-apoptotic $\mathrm{Bcl}-2$ like proteins ( $\mathrm{Bcl}-2, \mathrm{Bcl}-\mathrm{XL}$ and $\mathrm{Mcl}-1)$ is similarly downregulated by Sorafenib in hepatoma cells derived from C57L mice (Hepa1-6) and in primary hepatocytes from C57BL/6 mice. However, Sorafenib-mediated activation of caspase-3-dependent apoptosis was specifically found in hepatoma cells, but not in healthy hepatocytes. We validated these findings in vivo by applying an isograft HCC transplantation model and after $\mathrm{PH}$ in $\mathrm{C} 57 \mathrm{BL} / 6$ mice. We provided evidence that Sorafenib activates caspase-3 and thus apoptosis selectively in small tumor foci that originated from implanted Hepa1-6 cells but not in surrounding healthy hepatocytes. Similarly, Sorafenib did not induce apoptosis after $\mathrm{PH}$. However, Sorafenib treatment transiently inhibited cell cycle progression and resulted in incorrect mitosis and enhanced non-apoptotic liver injury during liver regeneration.

Previous studies already demonstrated that Sorafenib efficiently blocks cell cycle initiation in human HCC cell lines by inhibiting Raf/ERK signaling and Cyclin D induction. ${ }^{6}$ In our present study we extended these analyses in the murine model and provide new evidence demonstrating that these cell cycle inhibitory effects are a general property of Sorafenib and apply not only for hepatoma cells but also for mitogen-activated healthy hepatocytes in vitro and in vivo. We also addressed the regulatory mechanisms of Sorafenib on downstream G1 and S-phase cyclins, respectively. While Sorafenib downregulated Cyclins $D$ and A predominantly at the protein level, it also showed very strong and fast effects on Cyclin E1 gene transcription. We recently demonstrated that Cyclin E1 is essential for the activation and survival of hepatic stellate cells and induction of liver fibrosis, ${ }^{19}$ while its role for liver regeneration in hepatocytes can be replaced by overlapping Cyclins such as Cyclin A2. ${ }^{20}$ Thus, application of Sorafenib in patients with chronic liver disease could also act as an anti-fibrotic as suggested earlier. ${ }^{21}$ We further conclude that Sorafenib similarly mediates cell cycle arrest in malignant and non-malignant cells such as regenerating healthy hepatocytes via identical mechanisms. This has to be taken into account when applying Sorafenib to patients with advanced cirrhosis as residual liver function in these patients relies on regenerating hepatocytes.

Our finding that Sorafenib specifically activates caspase-3 and apoptosis in malignant hepatoma cells but not in non-malignant hepatocytes under any tested condition in vitro or in vivo is a key result of our study and defines a strong tumor-specific effect of Sorafenib. Our data are in good agreement with a previous paper demonstrating that Sorafenib even protects primary murine hepatocytes from TGF- $\beta$-induced apoptosis in vitro. ${ }^{22}$ However, the mechanism preventing Sorafenib-mediated apoptosis in healthy hepatocytes remains obscure and needs further investigation. The observed downregulation of the antiapoptotic Bcl-2-like proteins $\mathrm{Mcl}-1$ and $\mathrm{Bcl}-\mathrm{XL}$ after Sorafenib treatment is not sufficient to explain caspase- 3 activation and apoptosis in hepatoma cells in vitro for two reasons. First, Mcl1 and $\mathrm{Bcl}-\mathrm{XL}$ were also downregulated by Sorafenib in primary hepatocytes that lacked caspase-3 activation. Second, previous studies suggested that spontaneous liver apoptosis after genetic inhibition of Mcl-1 and/or Bcl-XI is most likely caused by latent expression of extrinsic death ligands such as Fas. ${ }^{23,24}$ It has been reported that Sorafenib induces apoptosis via PUMA in a p53-independent manner at least in colon carcinoma cells. ${ }^{15}$ In addition, PUMA is downregulated in liver after $\mathrm{PH}^{25}$ Our own data showed robust PUMA protein expression in hepatoma cells with or without Sorafenib treatment while PUMA remained at low level in primary hepatocytes in vitro and was even downregulated in liver after $\mathrm{PH}$. It is therefore tempting to speculate that Sorafenib induces hepatoma cell apoptosis in a PUMA-dependent manner, while hepatocytes are protected from Sorafenib-mediated apoptosis if PUMA is downregulated or absent.

For our studies on Sorafenib in mice we dissolved the drug in an aqueous solution containing ethanol (8.75\%), based on the manufacturer's recommendation and similar to previous animal studies. ${ }^{6,26,27}$ Unexpectedly, control experiments using the solvent alone already had profound inhibitory effects on cell cycle progression in mice after $\mathrm{PH}$, which have not been addressed before. Since patients receive Sorafenib medication in tablets, these findings do not have an impact for human therapy. However, the strong effect of the ethanol/ chremophor solvent on liver regeneration and potentially also on tumor cell proliferation could require a re-evaluation of some previous data gained in murine models.

Our data on the impact of Sorafenib for liver regeneration in mice expands a recent study by Hora et al. ${ }^{26}$ While these authors demonstrated that Sorafenib administration after hepatectomy affected late liver regeneration, our own study was rather focused on early liver regeneration (48 h post $\mathrm{PH}$ ), liver injury and the underlying signal transduction pathways. We clearly demonstrate that Sorafenib cannot induce caspase-3-mediated apoptosis in liver after $\mathrm{PH}$. However, 
Sorafenib frequently caused aberrant mitosis reflecting signs of mitotic catastrophe as reviewed recently, which correlated with elevated serum transaminases and thus with increased liver injury. It has been suggested that mitotic catastrophe correlates with incomplete DNA synthesis and premature chromosome condensation that eventually may lead to apoptosis or necrotic-like cell death. ${ }^{28}$ Our data from $\mathrm{PH}$ experiments revealed that the number of total mitosis events is similar between the Sorafenib- and solvent-treated groups while protein levels of Cyclin B1 (driving mitosis) and Cyclin A2 (driving DNA synthesis) are substantially reduced in the Sorafenib-treated cohort. We therefore conclude that the strong mitogen stimulation after $\mathrm{PH}$ (by exocrine mediators such as TNF, EGF, HGF and insulin) in combination with stringent inhibition of downstream Cyclins (E, A and B) may result in faulty DNA replication and irregular mitosis. Future histological analysis of biopsies from patients undergoing Sorafenib therapy would be necessary to evaluate whether our findings are also of clinical relevance.

Taken together, our results highlight the benefit of Sorafenib for specifically eliminating malignant hepatoma cells through apoptosis presumably in a PUMA-dependent manner. However, our study also revealed that Sorafenib may impair liver regeneration by inducing enhanced mitotic failure of healthy hepatocytes and tissue injury, which identifies a yet unknown risk factor.

\section{Materials and Methods \\ Housing and treatment of mice. All animals were maintained in the animal facility of the University Hospital Aachen in a temperature-controlled room with 12-hour light/dark cycle and free access to food and water. Experimental animal procedures were approved by the authority for environment conservation and consumer protection of the state North Rhine-Westfalia (LANUV, Germany, AZ84-02.04.2011.A365). For the studies, WT mice in a C57BL/6 background were used. $\mathrm{PH}$ was performed as described earlier. ${ }^{29}$ For any indicated time point and condition, 3-11 mice of male gender at the age of 6-8 weeks were analyzed.}

Measurement of aminotransferase activity. Alanine aminotransferase (ALT) and aspartate aminotransferase (AST) activities were measured in serum from mice according to standard methods (UV test at $37^{\circ} \mathrm{C}$ ) using a Roche Modular preanalytics system (Roche, Grenzach, Germany).

Cell culture procedures. Hepa1-6 hepatoma cells were derived from C57L/J mice ${ }^{9}$ and obtained from the German Collection of Microorganisms and Cell Cultures (DSMZ, Braunschweig, Germany). Primary hepatocytes were isolated from C57BL/6 mice as described recently. ${ }^{30}$ All cells were cultured in Dulbecco's Modified Eagle's Medium (PAA, Pasching, Austria) with 10\% fetal calf serum at $37^{\circ} \mathrm{C}$ in a humidified atmosphere containing $5 \% \mathrm{CO}_{2}$. For mitogen stimulation, hepatocytes were treated with $10 \mathrm{ng} / \mathrm{ml}$ EGF (Sigma-Aldrich, St. Louis, MO, USA) and $0.02 \mathrm{U} / \mathrm{ml}$ Insulin (Novo Nordisk, Mainz, Germany).

Application of Sorafenib (Bay 54-9085) in vitro and in vivo. Sorafenib tyoslate was provided by Bayer HealthCare AG Pharmaceuticals (Berlin, Germany). For in vitro studies, Sorafenib was dissolved in DMSO. For in vivo application, Sorafenib was dissolved in an aqueous solution containing $8.75 \%$ ethanol and $12.5 \%$ Chremophor EL. Sorafenib or solvent were administrated to mice at a dosage of $100 \mathrm{mg} / \mathrm{kg}$ by gavage in a total volume of $200 \mu$ l.

Quantitative real-time PCR (qPCR). Total RNA from murine hepatoma cells, primary hepatocytes and liver tissues was isolated using the peqGOLD RNAPure Kit (Peqlab, Erlangen, Germany). Reverse transcription was performed using an Omniscript RT Kit (Qiagen, Hilden, Germany). Relative quantitative gene expression was measured via real-time PCR using a 7300 Real Time PCR System with SDS software 1.3.1 (Applied Biosystems, Foster City, CA, USA) and a SYBR
Green PCR Kit (Invitrogen, Carlsbad, CA, USA). Target gene expression was normalized by determining GAPDH expression as internal standard and calculated as fold induction in comparison to untreated controls. The primer sequences are given in Supplementary Table 1.

Liver histology, in situ tissue staining and immunoblot analysis. Standard tissue stainings and immunohistochemistry were performed in the Department of Pathology at the University Hospital Aachen and analyzed by an experienced pathologist (N.G.). For quantitative analysis of Hematoxylin \& Eosin (H\&E) and chloroacetate esterase (CAE)-stained sections, one high-power field (HPF) was defined as an area of $237 \mathrm{~mm}^{2}$ at a magnification of $\times 200$. For the determination of DNA synthesis (S-Phase) in Hepa1-6 cells, primary hepatocytes and the regenerating liver, incorporation of BrdU (Applichem, Cheshire, CT, USA) was analyzed as described recently. ${ }^{31}$ In cultured cells, BrdU was added to the cell culture medium at a concentration of $6 \mu \mathrm{g} / \mathrm{ml} 2 \mathrm{~h}$ before fixation. For the evaluation of cell cycle activity in situ liver cryosections of $5 \mu \mathrm{m}$ were stained with an anti-Ki-67 antibody. Stained microscopic images were acquired at magnifications of $\times 200$ with a Zeiss Axio Imager.Z1 microscope, Axiocam MRm and HRc cameras using Axiovision 4.8 software (all from Carl Zeiss, Inc., Oberkochen, Germany). Isolation of whole-cell proteins from cultured cells and liver tissue and subsequent western blot analysis was performed as described previously. ${ }^{32}$ Antibodies used for analysis are listed in Supplementary Table 2. GAPDH and $\beta$-actin were used as loading controls. For the detection of cleaved caspase-3 via immunoblot analysis in whole-cell extracts of isolated primary hepatocytes and livers, murine Hepa1-6 hepatoma cells were treated with $50 \mu \mathrm{m}$ Sorafenib for $24 \mathrm{~h}$ and were used as a positive control (pos.). Immunoblots were visualized by Amersham ECL Prime (GE Healthcare) using the Fuji LAS-4000 Mini, according to the manufacturers' protocol.

Fluorescence-activated cell sorting of Hepa1-6 cells. Cultivated Hepa1-6 cells were harvested with Trypsin (PAN Biotech, Aidenbach, Germany) after $24 \mathrm{~h}$, washed with phosphate-buffered saline and fixated with $4 \%$ paraformaldehyde for $15 \mathrm{~min}$. After fixation cells were permeabilized in Nicoletti Buffer $(0.1 \%$ sodium citrate, $0.1 \%$ Triton 100$)$ for $20 \mathrm{~min}$, stained with PI/RNASE staining Buffer (BD Bioscience, Heidelberg, Germany) and analyzed on a FACS Canto II Flowcytometer (BD). Data were analyzed using FlowJo 7.5 Software (Tree Star, Ashland, OR, USA)

Determination of apoptosis. In situ, apoptosis was determined on liver cryosections or on Hepa1-6 cells that adhered to coverslips using an In Situ Cell Death Detection Kit (Roche Diagnostics, Mannheim, Germany) according to manufacturer's instructions. Alternatively, apoptosis was determined by quantification of specific caspase-3 enzyme activity (fluorescence units/ $\mu \mathrm{g}$ protein) in protein lysates from cultured cells as described recently. ${ }^{33}$ In brief, protein lysates were incubated with the artificial substrate AFC-DEVD (Enzo Life Sciences, Farmingdale, NY, USA) and the caspase-3 mediated release of AFC from DEVD was quantified by UV spectrometry. For determination of cleaved caspase-3 activity in whole-cell extracts of isolated primary hepatocytes and livers, murine Hepa1-6 hepatoma cells were treated with $50 \mu \mathrm{m}$ Sorafenib for $24 \mathrm{~h}$ and were used as a positive control (pos.)

Statistical analysis. Data were presented as mean \pm standard deviation of the mean. Statistical significance was determined by Student's $t$-test.

\section{Conflict of Interest}

The authors declare no conflict of interest.

Acknowledgements. This work was supported by the Deutsche Forschungsgemeinschaft (LI1045/2-4). We thank Bayer HealthCare Pharmaceuticals for providing Sorafenib.

\section{Author contributions}

Roland Sonntag: acquisition of data, analysis of data and drafting of the manuscript; Nikolaus Gassler: analysis of data; Jörg-Martin Bangen: acquisition and analysis of data. Christian Trautwein: analysis and interpretation of data, and drafting of the manuscript. Christian Liedtke: study design, study supervision, conception and 
composition of manuscript, and obtained funding. All authors read and approved the final version of the manuscript.

1. Marra M, Sordelli IM, Lombardi A, Lamberti M, Tarantino L, Giudice A et al. Molecular targets and oxidative stress biomarkers in hepatocellular carcinoma: an overview. J Trans Med 2011; 9: 171.

2. Montella L, Addeo R, Caraglia M, Del Prete S. Latest developments in targeted therapy for hepatocellular carcinoma. Exp Rev Anticancer Ther 2010; 10: 1635-1646.

3. Llovet JM, Ricci S, Mazzaferro V, Hilgard P, Gane E, Blanc JF et al. Sorafenib in advanced hepatocellular carcinoma. N Engl J Med 2008; 359: 378-390.

4. Llovet JM, Bruix J. Molecular targeted therapies in hepatocellular carcinoma. Hepatology 2008; 48: 1312-1327.

5. Wilhelm S, Carter C, Lynch M, Lowinger T, Dumas J, Smith RA et al. Discovery and development of sorafenib: a multikinase inhibitor for treating cancer. Nat Rev Drug Discov 2006; 5: 835-844.

6. Liu L, Cao Y, Chen C, Zhang X, McNabola A, Wilkie D et al. Sorafenib blocks the RAF/MEK/ERK pathway, inhibits tumor angiogenesis, and induces tumor cell apoptosis in hepatocellular carcinoma model PLC/PRF/5. Cancer Res 2006; 66: 11851-11858.

7. Bouillet P, Purton JF, Godfrey DI, Zhang LC, Coultas L, Puthalakath H et al. BH3-only Bcl-2 family member Bim is required for apoptosis of autoreactive thymocytes. Nature 2002; 415 : 922-926.

8. Yu C, Bruzek LM, Meng XW, Gores GJ, Carter CA, Kaufmann SH et al. The role of Mcl-1 downregulation in the proapoptotic activity of the multikinase inhibitor BAY 43-9006. Oncogene 2005; 24: 6861-6869.

9. Darlington GJ, Bernhard HP, Miller RA. Ruddle FH. Expression of liver phenotypes in cultured mouse hepatoma cells. J Natl Cancer Inst 1980; 64: 809-819.

10. Downward J. PI 3-kinase, Akt and cell survival. Semin Cell Dev Biol 2004; 15: 177-182.

11. Hu W, Nevzorova YA, Haas U, Moro N, Sicinski P, Geng Y et al. Concurrent deletion of cyclin e1 and cyclin-dependent kinase 2 in hepatocytes inhibits DNA replication and liver regeneration in mice. Hepatology 2013; e-pub ahead of print 24 December 2013; doi:10.1002/hep.26584

12. Scholzen T, Gerdes J. The Ki-67 protein: from the known and the unknown. J Cell Physiol 2000; 182: 311-322.

13. Diril MK, Ratnacaram CK, Padmakumar VC, Du T, Wasser M, Coppola V et al. Cyclindependent kinase 1 (Cdk1) is essential for cell division and suppression of DNA re-replication but not for liver regeneration. Proc Natl Acad Sci USA 2012; 109: 3826-3831.

14. Miyaoka Y, Ebato K, Kato H, Arakawa S, Shimizu S, Miyajima A. Hypertrophy and unconventional cell division of hepatocytes underlie liver regeneration. Curr Biol 2012; 22 1166-1175.

15. Dudgeon C, Peng R, Wang P, Sebastiani A, Yu J, Zhang L. Inhibiting oncogenic signaling by sorafenib activates PUMA via GSK3beta and NF-kappaB to suppress tumor cell growth. Oncogene 2012; 31: 4848-4858.

16. Xie B, Wang DH, Spechler SJ. Sorafenib for treatment of hepatocellular carcinoma: a systematic review. Digest Dis Sci 2012; 57: 1122-1129.

17. Santini D, Addeo R, Vincenzi B, Calvieri A, Montella L, Silletta M et al. Exploring the efficacy and safety of single-agent sorafenib in a cohort of Italian patients with hepatocellular carcinoma. Exp Rev Anticancer Ther 2012; 12: 1283-1288.

18. Xu M, Xie XH, Xie XY, Xu ZF, Liu GJ, Zheng YL et al. Sorafenib suppresses the rapid progress of hepatocellular carcinoma after insufficient radiofrequency ablation therapy: an experiment in vivo. Acta Radiol 2013; 54: 199-204.
19. Nevzorova YA, Bangen JM, Hu W, Haas U, Weiskirchen R, Gassler N et al. Cyclin E1 controls proliferation of hepatic stellate cells and is essential for liver fibrogenesis in mice. Hepatology 2012; 56: 1140-1149.

20. Nevzorova YA, Tschaharganeh D, Gassler N, Geng Y, Weiskirchen R, Sicinski P et al. Aberrant cell cycle progression and endoreplication in regenerating livers of mice that lack a single E-type cyclin. Gastroenterology 2009; 137: 691-703; 703 e691-696.

21. Wang $Y$, Gao J, Zhang D, Zhang J, Ma J, Jiang $H$. New insights into the antifibrotic effects of sorafenib on hepatic stellate cells and liver fibrosis. J Hepatol 2010; 53 132-144.

22. Chen YL, Lv J, Ye XL, Sun MY, Xu Q, Liu CH et al. Sorafenib inhibits transforming growth factor beta1-mediated epithelial-mesenchymal transition and apoptosis in mouse hepatocytes. Hepatology 2011; 53: 1708-1718.

23. Vick B, Weber A, Urbanik T, Maass T, Teufel A, Krammer PH et al. Knockout of myeloid cell leukemia-1 induces liver damage and increases apoptosis susceptibility of murine hepatocytes. Hepatology 2009; 49: 627-636.

24. Cazanave SC, Gores GJ. The liver's dance with death: two Bcl-2 guardian proteins from the abyss. Hepatology 2009; 50: 1009-1013.

25. Chen S, Zheng J, Hao Q, Yang S, Wang J, Chen $\mathrm{H}$ et al. p53-insensitive PUMA down-regulation is essential in the early phase of liver regeneration after partial hepatectomy in mice. J Hepatol 2010; 52: 864-871.

26. Hora $\mathrm{C}$, Romanque $\mathrm{P}$, Dufour JF. Effect of sorafenib on murine liver regeneration. Hepatology 2011; 53: 577-586.

27. Wilhelm SM, Carter C, Tang L, Wilkie D, McNabola A, Rong H et al. BAY 43-9006 exhibits broad spectrum oral antitumor activity and targets the RAF/MEK/ERK pathway and receptor tyrosine kinases involved in tumor progression and angiogenesis. Cancer Res 2004; 64: 7099-7109.

28. Vakifahmetoglu H, Olsson M, Zhivotovsky B. Death through a tragedy: mitotic catastrophe. Cell Death Differ 2008; 15: 1153-1162.

29. Wustefeld T, Rakemann T, Kubicka S, Manns MP, Trautwein C. Hyperstimulation with interleukin 6 inhibits cell cycle progression after hepatectomy in mice. Hepatology 2000; 32 514-522.

30. Pietrangelo A, Dierssen U, Valli L, Garuti C, Rump A, Corradini E et al. STAT3 is required for IL-6-gp130-dependent activation of hepcidin in vivo. Gastroenterology 2007; 132: 294-300.

31. Dierssen U, Beraza N, Lutz HH, Liedtke C, Ernst M, Wasmuth HE et al. Molecular dissection of gp130-dependent pathways in hepatocytes during liver regeneration. $J$ Biol Chem 2008; 283: 9886-9895.

32. Zschemisch NH, Liedtke C, Dierssen U, Nevzorova YA, Wustefeld T, Borlak J et al. Expression of a cyclin $\mathrm{E} 1$ isoform in mice is correlated with the quiescent cell cycle status of hepatocytes in vivo. Hepatology 2006; 44: 164-173.

33. Liedtke C, Lambertz D, Schnepel N, Trautwein C. Molecular mechanism of Mitomycin C-dependent caspase-8 regulation: implications for apoptosis and synergism with interferon-alpha signaling. Apoptosis 2007; 12: 2259-2270.

(c) (i) $($ ) $\odot$ Cell Death and Disease is an open-access journal published by Nature Publishing Group. This work is licensed under a Creative Commons Attribution-NonCommercialNoDerivs 3.0 Unported License. To view a copy of this license, visit http://creativecommons.org/licenses/by-nc-nd/3.0/ 